\title{
IMPROVEMENTS IN DIRECT LYAPUNOV STABILIZATION OF UNDERACTUATED MECHANICAL SYSTEMS BY MEANS OF THE SOLVABILITY OF THE FIRST MATCHING CONDITION.
}

\author{
DEYKA GARCÍA and MARCELO CORONADO \\ Mechanical Engineering Department at the Universidad Tecnologica de Panamá \\ Campus Victor Levi, Building 1, Floor 3. Panamá, Republica de Panamá \\ E-mail: deyka.garcia@utp.ac.pa, marcelo.coronado@utp.ac.pa \\ http://www.utp.ac.pal \\ ANTONY GARCÍA \\ Electrical Engineering Laboratory at the Universidad Tecnologica de Panamá \\ Campus Victor Levi, Building 1, Floor 2. Panamá, Republica de Panamá \\ E-mail: antony.garcia@utp.ac.pa
}

\begin{abstract}
A solvability of the direct Lyapunov first matching condition in terms of the generalized coordinates method is presented for the stabilization of underactuated, inverted pendulum cart. This work represents a continued development of previously published techniques for the exact controller design formulation without introducing control law terms or approximations. The use of the control law, instead of inverse dynamics to determine the coordinate histories for the unspecified axes, produced good performance results showing a faster response to stabilize both the actuated and non-actuated axes. The stability is achieved from the proper shape of the potential, the positive definiteness of the $\mathbf{K}_{\mathbf{D}}$ matrix, and the non-positive rate of change of the Lyapunov function.
\end{abstract}

\section{Introduction}

There are several different types of mechanical systems which can be termed as underactuated. The degrees of freedom (DOF) of a system are defined by the system's number of independent movements. Underactuated mechanical systems have fewer actuators than DOF. Some examples such as satellites, aircraft, overhead crane loads, and missiles have at least one non-actuated DOF. Furthermore, the work presented here develops a nonlinear control law $\tau$ for the asymptotic stabilization of underactuated systems. This is accomplished by finding the solution of matching conditions that arise from Lyapunov's second method, analogous to the dissipation of energy. The direct Lyapunov approach (DLA) offers a wide range of applications for underactuated systems since the algebraic equations, ordinary differential equations, and partial differential equations stemming from the matching conditions are more tractable than those appearing in other approaches. The DLA was first presented in [1] and was applied to the stabilization of a class of systems characterized by dynamic equations where the nonlinearities depended on only one generalized coordinate and generalized velocity. Notable contributions in the study of stabilizing underactuated mechanical systems have been made by [2], [3] and [4] with the DLA. Another recent study is the Solvability of the Direct Lyapunov First Matching Condition in Terms of the Generalized Coordinates (DLFMC) of [5] which also contain more extensive literature reviews. All these approaches rely on a matching equation solution method. The DLFMC provides a method to solve the first matching condition and shows the tools for the control law. The main concentration of the control design methods previously mentioned, as well as the focus of this paper, is the stabilization of the Underactuated Mechanical Systems. 
The present paper continues the development of the DLA with a controller design formulation, which satisfies the First Matching Condition (FMC) exactly without introducing control law terms that are quadratic in the velocities or approximations. Applying DLA to more complicated systems showed the Lyapunov function and its derivative are non-monotonic terms. This difficulty was addressed in [6] where if enough conditions are satisfied, a monotonic standard Lyapunov function depending on the dynamics of the uncertain system can be posteriorly constructed.

A better formulation of the problem addressed in [7] is presented to improve the stability criterion. The problem addressed in [8] is presented to achieve better performance, and a relaxed nonmonotonic.

This problem is addressed in [9] and [10] using techniques that solve the matching equations, such that the first matching condition is satisfied in terms of the generalized coordinates. Certain restrictions were implemented to develop a nonlinear stabilizing control law for the inverted pendulum cart, which improve the performance.

In this paper certain parameters will be introduced to preserve the proper shape of the potential, the positive definiteness of the matrix, and the non-positive rate of change of the Lyapunov function.

To illustrate the formulation, the derivation is performed using the symbolic manipulation program Maple and it is simulated in the Matlab/Simulink environment.

\section{The Lyapunov formulation and the matching conditions}

The derivation of the new controller follows, in many respects, that presented by [2], [3], and [4] resulting in three matching conditions. The solution determines the stabilizing controller. Once the solution is found, the parameters involved in the matching equations are selected in terms of the stabilization and performance.

The FMC is used for the determination of the matrix. The goal in solving the FMC is to find the matrix that plays a role in both second and third matching conditions, such that the matrix is symmetric and positive definite together with the control input.

The Lyapunov stability theory is the main tool used in the analysis and synthesis of this development. The Lyapunov candidate function is defined in (2). The control input that satisfies the time derivative constraints of the Lyapunov candidate function along the system trajectories is designed such that $\dot{\boldsymbol{V}}(\mathbf{q}, \dot{\mathbf{q}}) \leq 0, \boldsymbol{V}(\mathbf{q}, \dot{\mathbf{q}})$ decreases to a constant, and $\mathbf{K}_{\mathbf{D}}=\mathbf{K}_{\mathbf{D}}{ }^{\mathbf{T}}>0$. The dynamic equations contain of the positive definite inertia matrix $\mathbf{M}(\mathbf{q}) \in \mathfrak{R}^{n \times n}$, a matrix $\mathbf{C}(\mathbf{q}, \dot{\mathbf{q}})$ $\in \mathfrak{R}^{n x n}$ containing the Coriolis and centripetal coefficients, and the vector $\mathbf{G}(\mathbf{q}) \in \mathfrak{R}^{n}$ consisting of forces and/or moments stemming from gradients of conservative fields. The equations of motion are

$$
\mathbf{M}(\mathbf{q}) \ddot{\mathbf{q}}+\mathbf{C}(\mathbf{q}, \dot{\mathbf{q}}) \dot{\mathbf{q}}+\mathbf{G}(\mathbf{q})=\left[\begin{array}{l}
\tau \\
0
\end{array}\right]
$$

The candidate Lyapunov function is stated as

$$
V(\mathbf{q}, \dot{\mathbf{q}})=\frac{1}{2} \dot{\mathbf{q}}^{\mathrm{T}} \mathbf{K}_{\mathbf{D}} \dot{\mathbf{q}}+\Phi(\mathbf{q})
$$

$\Phi(\mathbf{q}): \mathfrak{R}^{n} \rightarrow \mathfrak{R}$ is a potential function, and $\mathbf{K}_{\mathbf{D}} \in \mathfrak{R}^{n \times n}$ is a symmetric, positive definite matrix defined as

$$
\mathbf{K}_{\mathbf{D}}=\mathbf{P}(\mathbf{t}) \mathbf{M}(\mathbf{q})
$$


where $\mathbf{P}(\mathrm{t}) \in \mathfrak{R}^{n \times n}$ is a matrix chosen such that $\mathbf{K}_{\mathbf{D}}$ has the stated properties. Lyapunov's equation can be used to show that all the eigenvalues of $\mathbf{P}$ have positive real parts. This important result will be used in the second matching condition.

The time derivative of the candidate Lyapunov function is computed as

$$
\dot{V}=\dot{\mathbf{q}}^{\mathrm{T}} \mathbf{K}_{\mathrm{D}} \ddot{\mathbf{q}}+\frac{1}{2} \dot{\mathbf{q}}^{\mathrm{T}} \dot{\mathbf{K}}_{\mathrm{D}} \dot{\mathbf{q}}+\dot{\mathbf{q}}^{\mathrm{T}} \nabla \Phi(\mathbf{q})=-\dot{\mathbf{q}}^{\mathrm{T}} \mathbf{K}_{\mathrm{V}} \dot{\mathbf{q}}
$$

where the matrix $\mathbf{K}_{\mathbf{v}} \in \mathfrak{R}^{n \times n}$ is symmetric and at least positive semi-definite and $\boldsymbol{\nabla} \Phi(\boldsymbol{q})$ is the gradient of the potential with respect to the generalized positions. Owing to the nature of the right hand side of (4), LaSalle's theorem will be necessary to demonstrate asymptotic stability, however the right side of (4) is similar to the Hamiltonian formulations cited earlier. The result in (4) shows that $\dot{V}(\mathbf{q}, \dot{\mathbf{q}})$ is a non-positive function. Substituting for $\ddot{\mathbf{q}}$ from (1) into (3) produces

$$
\begin{aligned}
\dot{\mathrm{V}}= & \dot{\mathbf{q}}^{\mathrm{T}} \mathbf{K}_{\mathrm{D}} \mathbf{M}(\mathbf{q})^{-1}((-\mathbf{C}(\mathbf{q}, \dot{\mathbf{q}})) \dot{\mathbf{q}}-\mathbf{G}(\mathbf{q})+\boldsymbol{\tau}) \\
& +\frac{1}{2} \dot{\mathbf{q}}^{\mathrm{T}} \dot{\mathbf{K}}_{\mathrm{D}} \dot{\mathbf{q}}+\dot{\mathbf{q}}^{\mathrm{T}} \nabla \Phi\left(\mathbf{q}_{\mathrm{d}}\right)=-\dot{\mathbf{q}}^{\mathrm{T}} \mathbf{K}_{\mathrm{v}} \dot{\mathbf{q}} .
\end{aligned}
$$

The strategy in solving (5) is through a matching equation approach by breaking (5) into three separate equations. The three matching conditions are developed in the coming sections.

Examination of (5) shows there are two classes of terms that occur excluding the input. The first involves those terms that are pre and post multiplied by the generalized velocities. The other terms are pre-multiplied by a generalized velocity and involve a vector function of generalized coordinates (gravity terms and potential gradient) and these terms will give rise to the third matching condition. The first class of terms can be further divided into terms that are a function of the generalized velocities such as $\mathbf{C}(\mathbf{q}, \dot{\mathbf{q}})$ (these terms are found in the first matching condition) and terms that are constant such as $\mathbf{K}_{\mathrm{v}}$ (terms comprising the second matching condition). The input vector $\tau$ will be broken into three parts, one for each matching condition. Following these descriptions, (5) is written as three separate equations or matching conditions. The first matching condition is

$$
\dot{\mathbf{q}}^{\mathrm{T}} \mathbf{K}_{\mathrm{D}} \mathbf{M}(\mathbf{q})^{-1}\left(-\mathbf{C}(\mathbf{q}, \dot{\mathbf{q}}) \dot{\mathbf{q}}+\left[\begin{array}{c}
\mathbf{F}_{1} \\
0
\end{array}\right]\right)+\frac{1}{2} \dot{\mathbf{q}}^{\mathrm{T}} \dot{\mathbf{K}}_{\mathrm{D}} \dot{\mathbf{q}}=0
$$

The second matching condition is given by

$$
\dot{\mathbf{q}}^{\mathrm{T}} \mathbf{K}_{\mathrm{D}} \mathbf{M}(\mathbf{q})^{-1}\left(-\mathbf{C}_{\mathrm{D}} \dot{\mathbf{q}}+\left[\begin{array}{c}
\mathbf{F}_{2} \\
0
\end{array}\right]\right)=-\dot{\mathbf{q}}^{\mathrm{T}} \mathbf{K}_{\mathrm{v}} \dot{\mathbf{q}} .
$$

Finally, the third matching condition is provided by

$$
\dot{\mathbf{q}}^{\mathrm{T}} \mathbf{K}_{\mathrm{D}} \mathbf{M}(\mathbf{q})^{-1}\left(-\mathbf{G}(\mathbf{q})+\left[\begin{array}{c}
\mathbf{F}_{3} \\
0
\end{array}\right]\right)+\dot{\mathbf{q}}^{\mathrm{T}} \nabla \Phi(\mathbf{q})=0 .
$$

By defining the first matching condition control input as.

$$
\frac{1}{2} \dot{\mathbf{K}}_{\mathrm{D}}+\mathbf{K}_{\mathrm{D}} \mathbf{M}(\mathbf{q})^{-1}\left(\left[\begin{array}{c}
\mathbf{F m}_{\mathbf{1}} \\
0
\end{array}\right]-\mathbf{C}(\mathbf{q}, \dot{\mathbf{q}})\right)=0 .
$$

where $\mathbf{F m}_{1} \in \mathfrak{R}^{m \times n}$ is a coefficient matrix yet to be determined. Each term in the first matching condition is pre and post multiplied by the vector of generalized velocities. 
The symmetric part of (9) to zero produces

$$
\dot{\mathbf{K}}_{\mathrm{D}}+\mathbf{K}_{\mathrm{D}} \mathbf{M}(\mathbf{q})^{-1}\left(\left[\begin{array}{c}
\mathbf{F m}_{\mathbf{1}} \\
0
\end{array}\right]-\mathbf{C}(\mathbf{q}, \dot{\mathbf{q}})\right)+\left(\left[\begin{array}{c}
\mathbf{F} \mathbf{m}_{1} \\
0
\end{array}\right]-\mathbf{C}(\mathbf{q}, \dot{\mathbf{q}})\right)^{\mathrm{T}} \mathbf{M}(\mathbf{q})^{-1} \mathbf{K}_{\mathrm{D}}=0
$$

\subsection{The First Matching Condition}

The goal in solving the first matching condition is the determination of both the matrix $\mathbf{K}_{\mathbf{D}}$ such that the matrix is symmetric and positive definite and the control input $F_{1}$. Likewise, the goal of the second matching condition solution is finding the matrix $\mathbf{K}_{\mathrm{v}}$ such that it is symmetric and at least positive semi-definite and the control law contribution $F_{2}$. The overall goal in satisfying the third matching condition is the determination of the potential $\Phi$ and the final portion of $\tau$, namely $F_{3}$.

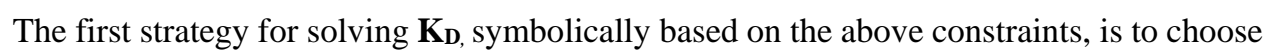
the elements of Fm 1 and Fmc 1 as non-zero in (6) which result is showed in (10). In order to satisfy (10), $n(n+1) / 2$ equations can be written to determine the same number of unknowns.

If $\mathbf{K}_{\mathrm{D}}$ is constant, then the matrix $\mathbf{P}$ shown in (3) becomes a function of $\mathbf{q}$ alone as seen by

$$
\mathbf{P}(\mathbf{q})=\mathbf{K}_{\mathbf{D}} \mathbf{M}(\mathbf{q})^{-1}
$$

where $\mathbf{P}(\mathbf{q})$ is the matrix that will have a significant role in the definition of the main controller characteristics and the potential $\Phi$. It is essential to have a full, non-singular or at least lower triangular $\mathbf{P}(\mathbf{q})$ matrix to achieve this because the first $m$ columns of the $\mathbf{P}(\mathbf{q})$ matrix are used to determine the $\mathbf{K}_{\mathbf{v}}$ matrix in the Second Matching Condition.

\subsection{The Second matching condition}

This section will present the solution of the second matching condition. Let the forcing term of (7) be written as

$$
\left[\begin{array}{c}
\mathbf{F}_{2} \\
\mathbf{0}
\end{array}\right]=\left[\begin{array}{c}
\mathbf{F m}_{2} \\
\mathbf{0}
\end{array}\right] \dot{\boldsymbol{q}}
$$

where $\mathbf{F m}_{2}$ is a $m \times n$ real matrix. Substituting (12) into (7), removing the pre and post multiplication by the generalized velocity vector, and simplifying shows

$$
\mathbf{P}(\mathbf{q})\left[\begin{array}{c}
\mathbf{F} \mathbf{m}_{2} \\
\mathbf{0}
\end{array}\right]=-\mathbf{K}_{v} .
$$

The condition that $\mathbf{K}_{\mathbf{v}}$ be symmetric provides $n(n-1) / 2$ linear algebra equations. In order to solve for the matrix $\mathbf{F m} 2$, (13) is multiply by $\mathbf{P}(\mathbf{q})^{-\mathbf{1}}$ to get

$$
\left[\begin{array}{c}
\boldsymbol{F} m_{2} \\
0
\end{array}\right]_{1}=-\mathbf{P}(\mathbf{q})^{-1} \mathbf{K}_{\mathrm{v}}
$$

for which the solution is

$$
\mathbf{K}_{\mathrm{v}}=\sum_{\mathrm{i}=1}^{\mathrm{m}} \alpha_{i} \mathbf{P}_{\mathrm{i}} \mathbf{P}_{\mathrm{i}}^{\mathrm{T}}
$$

where the $\alpha_{i}$ are constants chosen so that $\mathbf{K}_{\mathrm{v}}$ is positive semi-definite and $\mathbf{P}_{\mathbf{i}}$ is the $\mathrm{i}^{\text {th }}$ column of $\mathbf{P}(\mathbf{q})$. Note that the matrix on the left side of (14) (part of the control law) is evaluated by extraction of the first $m$ rows of the matrix product on the right side of (14). 
Because $\mathbf{K}_{\mathbf{v}}$ needs to be at least positive- semi definite, the eigenvalues of $\mathbf{K}_{\mathbf{v}}$ are required to be

$$
\operatorname{eig}\left(\mathbf{K}_{v}\right) \geq 0
$$

\subsection{The Third matching condition}

In order to solve the third matching condition, the first matching equation must be solved, because the $\mathbf{P}(\mathbf{q})$ matrix is needed. From (8), the third matching equation is stated as

$$
-\mathbf{P}(\mathrm{t}) \mathbf{G}(\mathbf{q})+\mathbf{P}(\mathrm{t})\left[\begin{array}{c}
\mathbf{F}_{3} \\
0
\end{array}\right]+\nabla \Phi(\mathbf{q})=0
$$

The first $m$ equations in (17) are used to determine the control law contribution $F_{3}$ while the last $n-m$ rows of the equation provide linear, first order partial (ordinary) differential equations for the potential $\Phi$.

In taking the time derivative of the candidate Lyapunov function, the potential $\Phi$ is assumed to be a function of the generalized positions $\mathbf{q}$ alone. In examining (17), it is seen that $\mathbf{P}(\mathrm{t})$ appears in the equation leading to the conclusion that $\Phi$ also depends upon $\mathbf{P}(\mathbf{t})$. If $\mathbf{K}_{\mathbf{D}}$ is constant then (11) applies and $\mathbf{P}$ is a function of the inverse of the mass matrix, which means that it is a function of $\mathbf{q}$ alone. The potential $\Phi(\boldsymbol{q})$ is also needed to assure the stability condition of the system. The Hessian of the potential denotes the second derivative of the potential with respect to the generalized coordinates. The determinant of the Hessian of the potential evaluated at $\mathrm{q}=0$ must be a positive. The necessary condition on $\mathrm{H}$ is

$$
\operatorname{eig}(\mathbf{H})>0 \text {. }
$$

The method to solve the third matching equation is based on the matching equations developed for stabilization as shown in [2], [3], and [4].

\section{The solution of $K_{D}$}

The equations of motion can be expressed as

$$
\mathbf{M} \ddot{\mathbf{q}}+(\mathbf{C}) \dot{\mathbf{q}}+\mathbf{G}=\mathbf{F}_{1} \dot{\mathbf{q}}+\mathbf{F}_{2}+\mathbf{F}_{3}
$$

where the three control law terms have been written in a more compact format. As before, the lower $n-m$ rows of the control law vectors are zero. Rearranging (4) and (10) the first matching condition is obtained

$$
\dot{\mathbf{K}}_{D}+\mathbf{K}_{D} \mathbf{M}(\mathbf{q})^{-1}\left(\mathbf{F} m_{1}-\mathbf{C}(\mathbf{q}, \dot{\mathbf{q}})\right)+\left(\mathbf{F} m_{1}-\mathbf{C}(\mathbf{q}, \dot{\mathbf{q}})\right)^{T} \mathbf{M}(\mathbf{q})^{-1} \mathbf{K}_{D}+\mathbf{F} m c_{1}=0 .
$$

The elements of the $\mathbf{K}_{\mathrm{D}}$ matrix are positive constants and the partial differential equation that needs to be solved for the potential is not complicated.

\section{Inverted pendulum cart (IPC) apparatus}

The control law design method was applied to an inverted pendulum cart system. The system geometry together with the dynamic equations of motion are shown in Figure 1 with definitions of the physical parameters. 


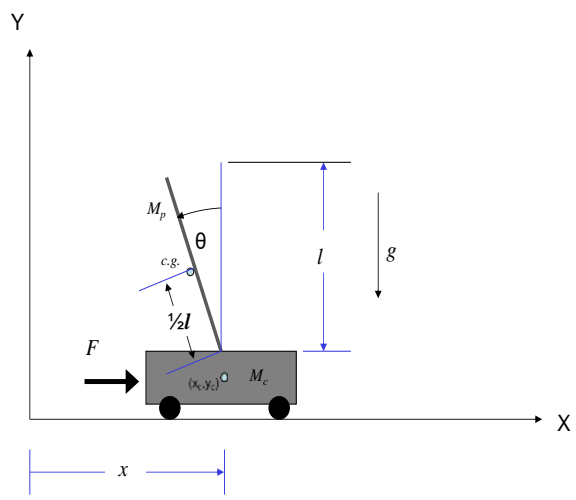

$$
\begin{aligned}
& {\left[\begin{array}{cc}
M_{c}+M_{p} & -\frac{1}{2} M_{p} l \cos (\theta) \\
-\frac{1}{2} M_{p} l \cos (\theta) & \frac{1}{4} M_{p} l^{2}+I_{p}
\end{array}\right]\left[\begin{array}{c}
\ddot{x} \\
\ddot{\theta}
\end{array}\right]+} \\
& {\left[\begin{array}{cc}
0 & -\frac{1}{2} M_{p} l \sin (\theta) \dot{\theta} \\
0 & 0
\end{array}\right]\left[\begin{array}{c}
\dot{x} \\
\dot{\theta}
\end{array}\right]+} \\
& {\left[\begin{array}{cc}
0 \\
-\frac{1}{2} M_{p} l \sin (\theta)
\end{array}\right]=\left[\begin{array}{c}
F \\
0
\end{array}\right]}
\end{aligned}
$$

Figure 1. Inverted Pendulum Cart System.

The values of the physical parameters are $l=0.7 \mathrm{~m}, g=9.81 \mathrm{~m} / \mathrm{s}^{2}, M_{c}=5 \mathrm{~kg}, M_{p}=1 \mathrm{~kg}$. $C_{l}=100, C_{2}=1$, are the constants values for the $\mathbf{K}_{\mathbf{D}}$ matrix , and $\alpha=38$ is a constant value for the Kv matrix.

\section{The simulation}

The chosen example could be stabilized by the nonlinear controller. The $\mathbf{K}_{\mathbf{D}}$ matrix was evaluated through numerical integration of (20).

The potential $\Phi(\mathrm{x}, \theta)$ for the inverted pendulum system is plotted on a positive interval for $\theta$, and it shows the concave shape, see Figure 2.

Figure 3 shows the Pendulum- Cart Positions and velocities.

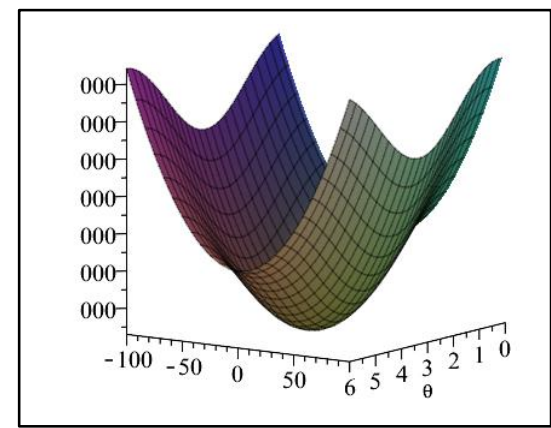

Figure 2. The potential for Inverted Pendulum Cart.
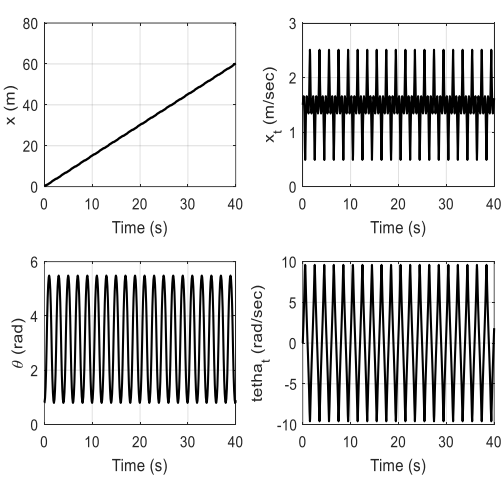

Figure 3. Pendulum- Cart Positions and velocities with no control law.

Figure 4 shows the control law, and Figure 5 shows the Pendulum- Cart Positions and velocities when stabilized. 


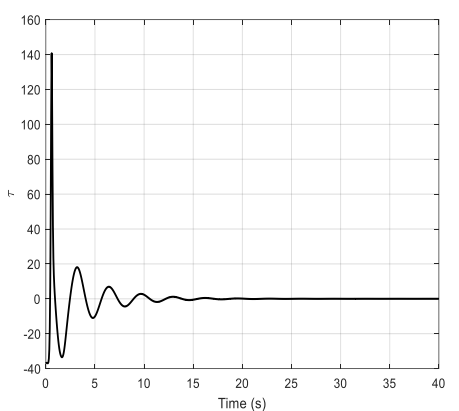

Figure 4: Control law
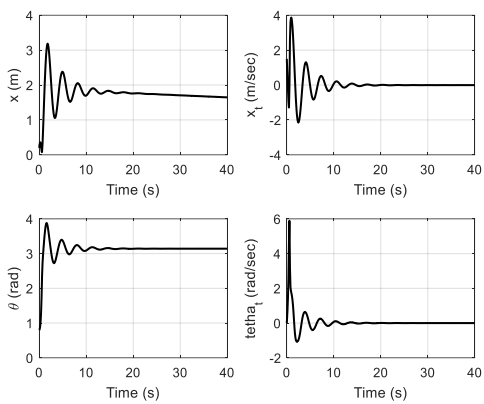

Figure 5: Pendulum- Cart Positions and velocities

Figure 6 shows the $\mathbf{P}$ matrix is either positive definite or positive semi-definite, and Figure 7 shows the elements of $\mathbf{P}$ are remaining essentially constant.

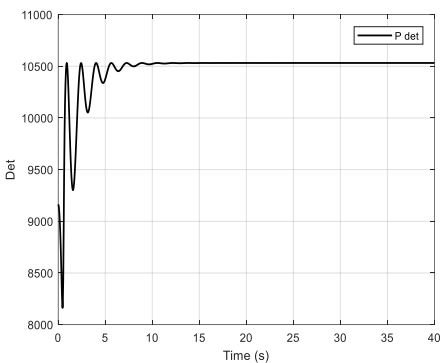

Figure 6: Determinant of P Matrix
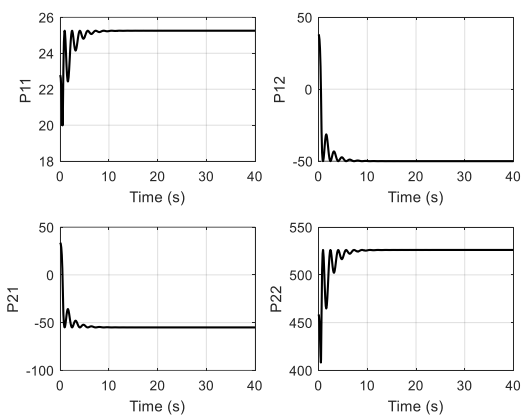

Figure 7: Elements of P Matrix

The behavior shown in Figure 8 demonstrates the validity of the Lyapunov candidate function.
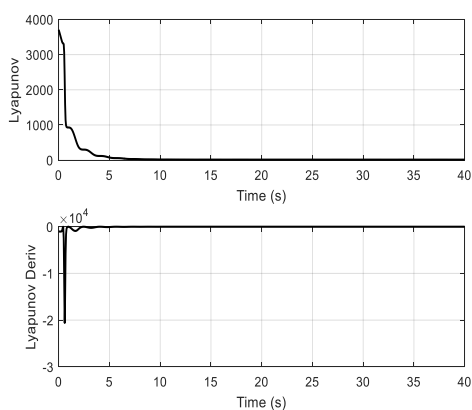

Figure 8: Lyapunov and Time Derivative History

\section{Conclusion}

A solvability of the direct Lyapunov first matching condition in terms of the generalized coordinates method for the stabilization of underactuated, inverted pendulum cart has been 
presented. The matching method, resulting in three equations, was improved to design the nonlinear system controller. The first matching condition results in PDEs for the positive and bounded for all time matrix $\mathbf{K}_{\mathbf{D}}$. Also, the eigenvalues of $\mathbf{P}(\mathbf{q})$ all have positive real parts. In this formulation, as was done in the previous one, $\mathbf{K}_{\mathbf{D}}$ approaches a constant matrix. In the previous formulation of the first matching equation, where several terms were implemented, the way in which the $\mathbf{K}_{\mathbf{D}}$ matrix was made to approach its final form could contribute to the $\mathbf{K}_{\mathbf{v}}$ matrix resulted in one eigenvalue of $\mathbf{K v}$ The present paper resulted in one eigenvalue of $\mathbf{K v}$ exhibiting a small oscillation when above or below an alpha value of one.

The second matching condition is formulated and solved in such a way that the Kv matrix always remains at least positive semi-definite.

The third matching condition produces $\mathrm{n}-\mathrm{m}$ linear PDEs for the potential $\Phi$. The third matching condition presents an expanded basin of attraction. In the example the expanded basin of attraction is determined by the choice of constants for $\mathbf{K}_{\mathbf{D}}$ and $\mathbf{K}_{\mathbf{V}}$.

All the simulation results obtained for the nonlinear systems validate the effective controller development in this work. The positive definiteness property of $\mathbf{K}_{\mathbf{D}}$, the proper shape of the potential, and the behavior of the Lyapunov time derivative were demonstrated.

\section{Acknowledgments}

The support of this work, in part, by the SENACYT Project No. ITE16R2IP006 is gratefully acknowledged.

\section{References}

1. W. N. White, M. Foss, and X. Guo, Am. Control Conf., 8 (2006).

2. W. N. White, M. Foss, and X. Guo, Proc. Am. Control Conf., No. Jan, 4817 (2007).

3. W. N. White, M. Foss, J. Patenaude, X. Guo, and D. García, Proc. American Control Conf., 2927 (2008).

4. W. N. White, J. Patenaude, M. Foss, and D. Garcia, American Control Conf., WeB20.4, 1341 (2009).

5. D. Garcia Batista, PhD Thesis, KSU, 387 (2012).

6. E. C. Zeeman, Nonlinearity, vol. 1, 15 (1988).

7. T. Tran, Int. Conf. Control. Autom. Inf. Sci. ICCAIS 2017, No. 1, 61 (2017).

8. M. J. Lacerda and P. Seiler, Automatica, vol. 82, 187 (2017).

9. D. García, I+D Tecnológico, vol. 10(2), 40 (2016).

10. D. I. García and W. N. White, Int. J. Agric. Biol. Eng., vol. 8(2), 72 (2014). 